\title{
Symptoms and Impacts in Non-Metastatic Castration-Resistant Prostate Cancer: Qualitative Study Findings
}

\author{
Erin L. Tomaszewski ${ }^{1} \cdot$ Pierre Moise $^{2} \cdot$ Robert N. Krupnick $^{3} \cdot$ Jared Downing $^{4} \cdot$

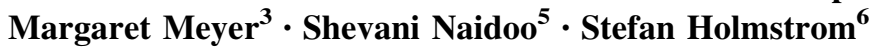

Published online: 11 March 2017

(c) The Author(s) 2017. This article is published with open access at Springerlink.com

\begin{abstract}
Objectives We developed a conceptual model to define key concepts associated with patients' experiences with the signs, symptoms, and impacts of non-metastatic castrationresistant prostate cancer (M0-CRPC).

Methods A targeted review of peer-reviewed literature, and other publicly available information, identified and categorized symptoms and impacts related to early-stage prostate cancer. Semi-structured interviews with five clinical experts helped determine the most relevant and important concepts for patients with M0-CRPC. Qualitative interviews with 17 patients with M0-CRPC identified the most frequently experienced symptoms and impacts, and their degree of interference with patients' lives. The findings from these three lines of evidence were summarized in a conceptual model.
\end{abstract}

Electronic supplementary material The online version of this article (doi:10.1007/s40271-017-0227-y) contains supplementary material, which is available to authorized users.

Pierre Moise

Pierre.Moise@quintiles.com

1 University of Pittsburgh, Pittsburgh, PA, USA

2 QuintilesIMS Inc., 3-5 rue Maurice Ravel, Levallois-Perret, 92594 Paris, France

3 QuintilesIMS Inc., Cambridge, MA, USA

4 QuintilesIMS Inc., Durham, NC, USA

5 Astellas Medical Affairs, Global HEOR, Chertsey, UK

6 Astellas Medical Affairs, Global HEOR, Leiden, The Netherlands
Results Literature searches identified mainly urinary, intestinal, and sexual symptoms. Experts noted the symptoms most frequently mentioned by patients include erectile dysfunction, loss of sexual desire or interest, incontinence/leaking, urgency, and hot flashes. Patient interviews confirmed the high frequency of erectile dysfunction, loss of libido, urinary urgency, and incontinence. The most frequently mentioned impacts expressed by patients were the need to monitor/plan for urinary frequency, interference with/restriction of daily activities, and frustration or anxiety over diagnosis, symptoms, or treatment. Symptoms and impacts most frequently experienced by patients were typically not those with the greatest effects on their lives; rather, those with the greatest consequences were related to treatment.

Conclusions The leading concerns associated with M0CRPC were related to voiding and sexual dysfunction. The most relevant symptoms and impacts expressed by patients may be a consequence of therapy rather than of the disease. 


\section{Key Points for Decision Makers}

In non-metastatic castration-resistant prostate cancer (M0-CRPC) there is limited information about disease symptoms, the patient's view of their impact, and patient-reported outcomes (PROs) most suitable for clinical studies. While, in aggregate, 'traditional' PRO tools are reasonably comprehensive for concepts previously identified in the literature, no single instrument sufficiently reflects the patient's experience of the effects of the disease and its treatment.

We developed a conceptual model that identifies the key aspects, from the patient's experience, of the signs, symptoms, and impacts of M0-CRPC. The model is based on a literature review, clinician interviews, and, most importantly, patient interviews. The most salient symptoms/impacts may be more related to treatment than disease, reflecting the patient's experience with therapy and luteinizing hormone-releasing hormone agonists.

This research may help identify the most important aspects of M0-CRPC to measure from the patient's perspective and lead to the development of more relevant PRO tools and outcome variables for this population.

\section{Introduction}

Non-metastatic castration-resistant prostate cancer (M0CRPC) is considered a relevant new target indication in the CRPC spectrum [1], which ranges from rising prostatespecific androgen (PSA) despite androgen deprivation therapy (ADT), without symptoms or metastases, to metastatic disease with significant debilitation [2]. While men with M0CRPC will likely have already undergone several treatments, their disease progresses relatively slowly [3].

The impact of prostate cancer (PCa) on health-related quality of life HR-QoL depends on disease stage and treatment [4]; HR-QoL continues to deteriorate as the disease progresses $[5,6]$. HR-QoL is a multi-domain concept that includes aspects of people's general perceptions of their physical, psychological, and social well-being [7]. Existing PCa-specific patient-reported outcome (PRO) instruments such as the Functional Assessment of Cancer Therapy-Prostate (FACT-P) [8] and the European Organisation for Research and Treatment of Cancer (EORTC) Quality of Life Questionnaire for Patients With Prostate
Cancer (QLQ-PR25) [9] were developed in the era before the evolution of the current definition of CRPC. While such instruments provide a broad perspective on HR-QoL for patients with M0-CRPC, they may not fully reflect all aspects of the effects of the disease and its treatment as experienced and reported by patients themselves.

When assessing the full impact of disease and treatment on HR-QoL, it is very important to consider the patient's perspective. Indeed, the European Medicines Agency and the US Food and Drug Administration have recently underscored the importance of the patient's voice in clinical trials $[7,10]$ by outlining steps for good PRO instrument development.

In M0-CRPC, there is a lack of information in the literature about the signs, symptoms, and impacts of the disease from the patient's perspective and specific recommendations on those PROs most suitable for clinical studies. Therefore, to understand patients' experiences of living with M0-CRPC, we sought to identify the most relevant and important signs, symptoms, and impacts of the disease in patients' experiences via a literature review, clinician interviews, and patient interviews.

There is broad agreement that PRO assessment should proceed from a strong conceptual basis regarding what is measured and how this is done [11]. A conceptual model helps specify the most important outcomes in a disease population and causal linkages and relationships among these outcomes [12]. Conceptual models are useful for identifying key endpoints for clinical studies and developing PROs to assess these endpoints [13]. We, therefore, aimed to develop a conceptual model to define key concepts associated with patients' experiences with the signs, symptoms, and impacts of M0-CRPC.

\section{Methods}

This qualitative patient interview study was conducted according to Parts 1 and 2 of the International Society for Pharmacoeconomics and Outcomes Research (ISPOR) PRO Good Research Practices Task Force Report [14, 15], which specify that selection of content for a PRO instrument involves synthesizing patient interviews, expert input, and conceptual disease-related information in the published literature [15]. Thus, a three-step process (literature review, clinician interviews, and patient interviews) identified signs and symptoms of M0-CRPC and the effects on daily life.

\subsection{Step 1: Literature Review}

Publically available information on symptoms and impacts of M0-CRPC were identified by (1) searching peer-reviewed literature in PubMed; (2) searching PCa research 
organizations, foundations, and patient blogs; (3) identifying and compiling common symptoms and impacts; and (4) categorizing overall disease concepts into the symptoms and impacts of PCa. This review was conceptual and designed to identify key articles describing the experience of PCa and/or PRO instruments in PCa. The PubMed search identified articles written in US English using different combinations of key words such as "prostate cancer", "PRO", "patient reported", and "quality of life". Abstracts were screened and full-text articles (and relevant references in these articles) that mentioned $\mathrm{PCa}$ and its signs, symptoms, and effects, or a PRO instrument for PCa, were retrieved and reviewed.

Based on the results of these searches, symptoms and symptom-related impacts of M0-CRPC were identified, compiled, and categorized. Overall, distal impacts related to the experience of having PCa were also included in the results of the searches.

\subsection{Step 2: Clinician Concept Elicitation Interviews}

Clinician interviews provided the clinical expert viewpoint on how patients experience the signs, symptoms, and impacts of M0-CRPC. These semi-structured telephone interviews included five US clinical experts familiar with management of M0-CRPC (oncologist, $n=2$; oncologist/ professor of surgery, $n=1$; urologist, $n=2$ ). Clinicians were identified from the literature. Those interested in participating were screened using a questionnaire that included number of years/location of practice, experience/duration treating patients with M0-CRPC, number of patients typically treated each month, and experience in patient-centric work. Physicians worked in major academic medical centers in the Northeast and Southeast $(n=3)$ or in private practice in the South and far West $(n=2)$. Clinicians received pre-interview background materials, including a description of objectives, summary of questions, and a preliminary conceptual model based on the literature review. Discussion was framed by a Clinician Interview Guide (see Electronic Supplementary Material), which was developed from the literature review, with a focus on specific signs, symptoms, and impacts associated with M0-CRPC. The guide started with open-ended questions about clinicians' perceptions of patients' experiences and ended with specific questions about anticipated treatment benefit. Concepts in the preliminary conceptual model were also reviewed for appropriateness for M0CRPC, relative perceived salience to patients, and possible omissions. Interviews (60-75 $\mathrm{min}$ ) were conducted by a trained interviewer with experience in concept elicitation. Interview notes were synthesized qualitatively, following standard market research procedures for qualitative content analysis.

\subsection{Step 3: Patient Concept Elicitation Interviews}

The research protocol and interview materials were approved by a central institutional review board. A digital process was followed to identify, screen, and recruit men with M0-CRPC. US-based members of MediGuard.org [16], a free online medication monitoring service owned and administered by Quintiles Transnational Holdings Inc. that has a database of 2.6 million patients, received an e-mail invitation to participate. Those accepting followed a link to a website with study information where they could provide consent to participate, self-screen for eligibility, and report baseline characteristics. Screening, demographic, and disease-related information were gathered directly from patients online, with no input from their physician(s).

The digital patient screening tool is summarized in Fig. 1. Eligible patients: were aged $>18$ years; had a diagnosis of $\mathrm{PCa}$, alone or combined with colorectal, lung, stomach, or pancreatic cancer, and without brain and/or bone metastases; had undergone surgical or medical castration using luteinizing hormone-releasing hormone (LHRH) or gonadotropin-releasing hormone $(\mathrm{GnRH})$ agonists; and had rising or stable PSA levels (since previous experience showed that the number of respondents could fall considerably following the second week of recruitment, the criterion of 'rising PSA' was relaxed to include patients with stable or unknown PSA levels). To avoid limiting our sample size, patients with PCa who also had other cancers were accepted. It was made clear to patients that interviews would focus only on PCa.

A semi-structured, qualitative Patient Interview Guide (see Electronic Supplementary Material) was developed from the literature review and clinician interviews. This guide included both open-ended and probing questions, and focused on symptoms and impacts of living with M0CRPC. Patients reported the frequency, severity, duration, and precipitating factors of symptoms/impacts, and how they disturbed their lives (on a scale of 0-10). We used the term 'symptoms' when asking respondents to focus on their day-to-day experience and asked them to characterize what they considered disease-specific or treatment-emergent symptoms; impacts focused more on the specific effects of these symptoms on patients' lives. Median interference scores were calculated for both symptoms and impacts.

Seventeen patients with M0-CRPC were interviewed (remotely via telephone and webcam) by a senior research professional experienced in patient interviewing and trained by senior staff/PRO experts experienced in PRO research. Symptoms, impacts, and interference scores were recorded on a Microsoft Excel ${ }^{\circledR}$ spreadsheet (Microsoft Corporation, Redmond, WA, USA).

By ordering the transcripts chronologically and dividing them into three groups according to the order of completion 


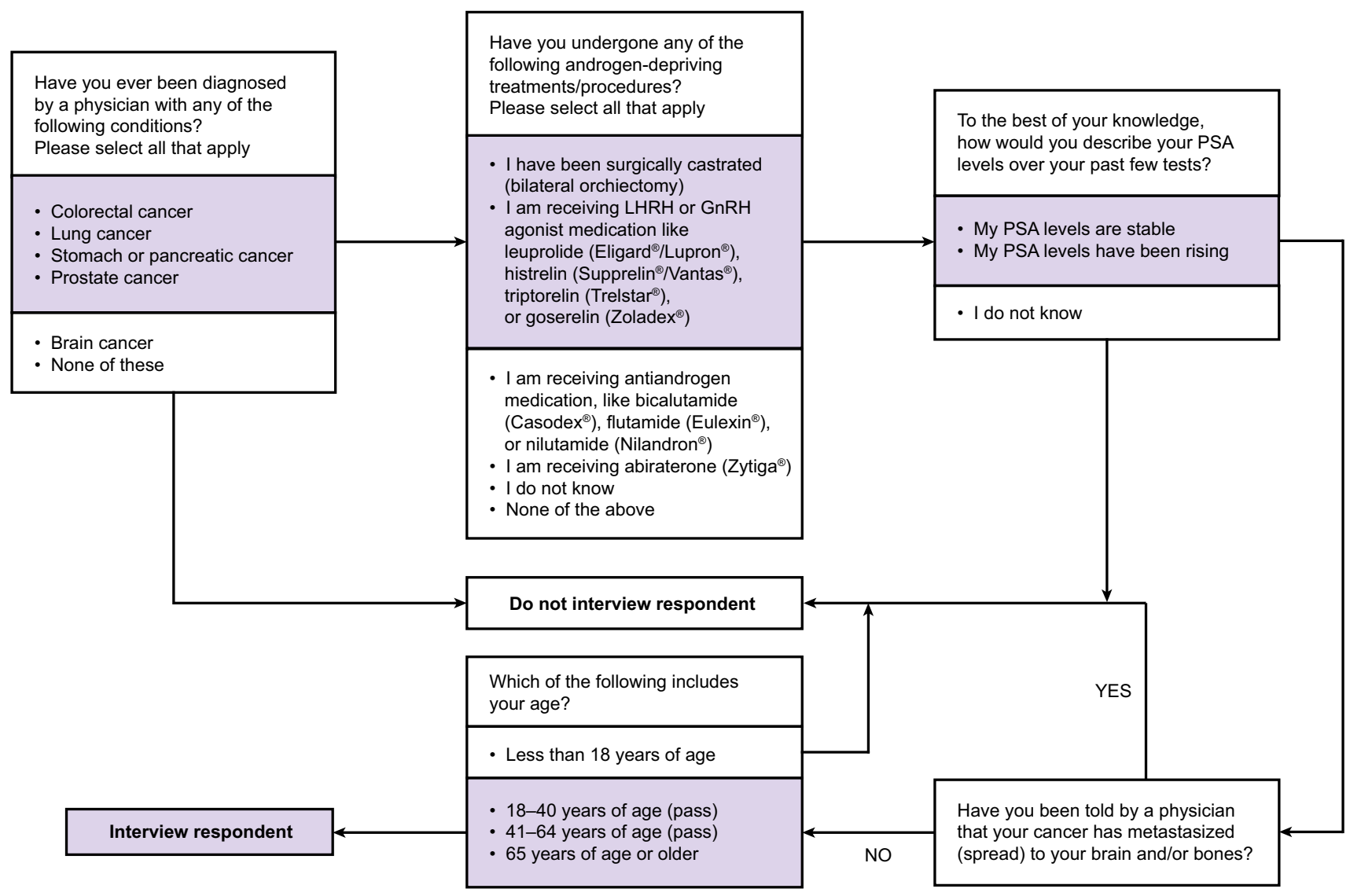

Fig. 1 Screening and recruitment process for identifying and enrolling men with non-metastatic castration-resistant prostate cancer. $G n R H$ gonadotropin-releasing hormone, $L H R H$ luteinizing hormone-releasing hormone, PSA prostate-specific antigen

(interviews 1-5, 6-10, and 11-17), a saturation table was prepared to ensure all relevant concepts were tracked. The most common concepts for each group were tabulated and compared with results from the previous group. Concept saturation was defined as no new occurrences in the last wave of interviews.

Concepts were included in the final model by combining information from three sources: the literature review, clinician interviews, and patient interviews. Patient selfreported information was weighted more than that from other sources. Concepts mentioned infrequently by patients were more likely to be included if corroborated by the other sources; otherwise, a minimum frequency of mention by three patients was set as a threshold for a concept to be included.

\subsection{Step 4: Qualitative Interview Analysis Methods}

As concept elicitation interviews were conducted, moderators noted responses live on printed symptom and impact recording worksheets. Transcripts were created to record verbatim what patients described after all interviews were conducted. All symptoms and impacts mentioned (both spontaneous and probed), and the corresponding interference scores, were recorded on a data analysis spreadsheet. If responses were unclear, transcripts were referenced to provide clarity. If a respondent described a symptom/impact in a similar fashion to that in the original conceptual model, it was grouped in the same response category. Researcher discretion was used to make the majority of these decisions. When categorization was unclear, the team made a collective decision about whether the symptom or impact should be counted separately, grouped with the original symptom/impact, or if the wording of the original symptom/impact should be changed. Number of mentions for each concept were summed to determine frequency of symptoms and impacts, and median ratings of interference for each concept were calculated.

\section{Results}

\subsection{Step 1: Literature Review}

In total, 16 articles [17-32] from the literature search were included in the review. The review revealed that M0-CRPC 


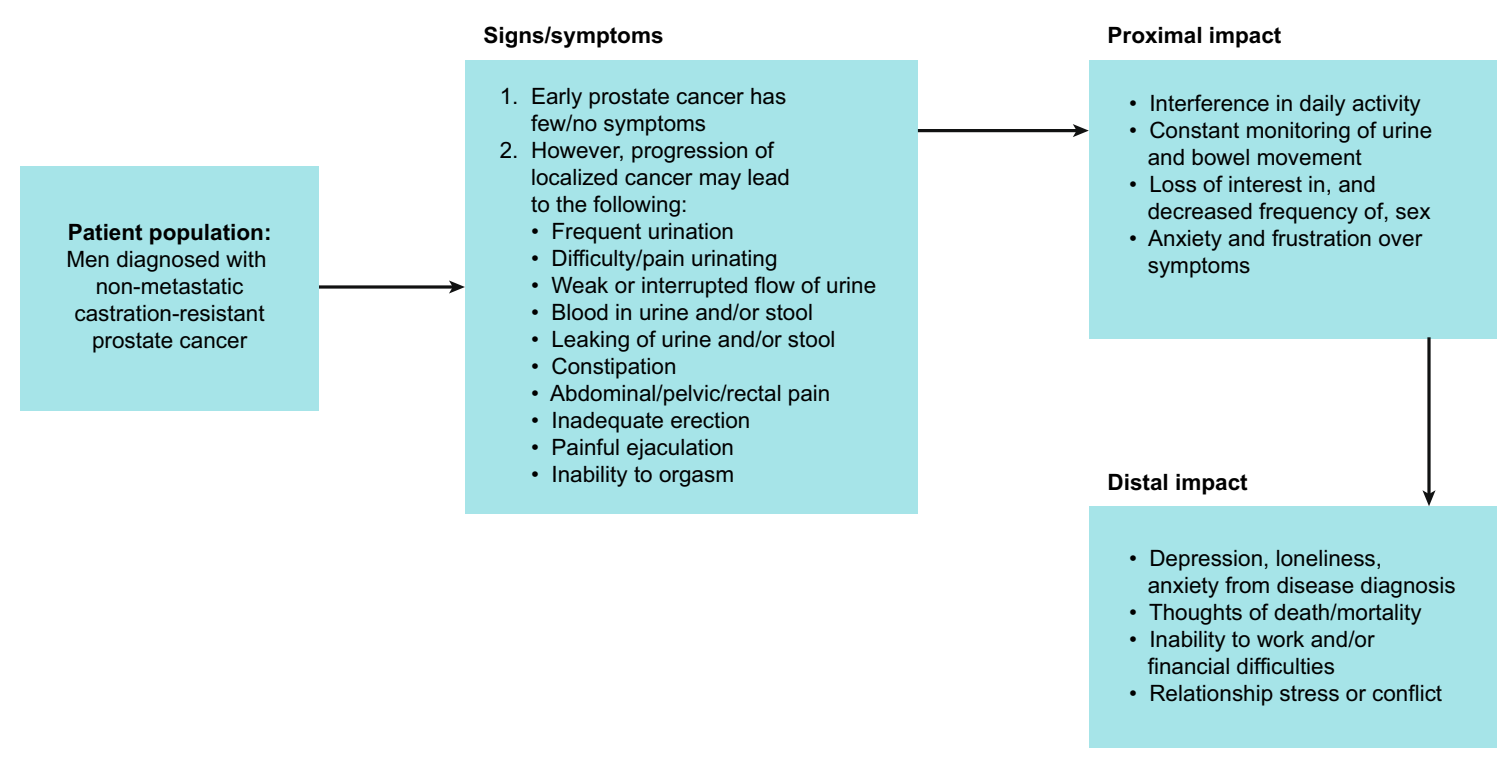

Fig. 2 Preliminary conceptual model (literature)

has few/no symptoms. A preliminary conceptual model was developed from this review and showed the most prominent symptoms and their impact on daily and overall life (see Fig. 2). Salient symptoms identified from literature searches were mainly urinary (frequent urination, difficulty/pain urinating, weak/interrupted flow of urine, blood in urine, leaking of urine), intestinal (constipation, abdominal/pelvic/rectal pain, blood in stools, leaking of stools), and sexual (inadequate erection, painful ejaculation, inability to achieve orgasm).

The main proximal impacts of symptoms on daily life were related to interference with daily activities, constant monitoring of urine and bowel movement, loss of interest in sex, decreased frequency of sex, and anxiety and frustration over symptoms. The more distal impacts of the disease were identified as depression, loneliness, and anxiety from carrying the disease diagnosis over time, thoughts of death/mortality, inability to work and/or financial difficulties, and relationship stress or conflict.

\subsection{Step 2: Clinician Concept Elicitation Interviews}

Experts confirmed several of the concepts most frequently mentioned by patients, including erectile dysfunction; loss of sexual desire or interest; incontinence/leaking; urgency; and hot flashes. All of these were endorsed by at least one expert as being attributed to prior treatments for $\mathrm{PCa}$, and all except hot flashes were attributed as important to patients they treat. All symptoms except incontinence/ leaking and urgency were attributed to ongoing treatments. Experts also noted that patients were anxious about rising PSA and the potential for disease progression and suggested that these be added to the conceptual model.
Some of the other concepts in the model were considered appropriate yet in need of modified terminology. Experts recommended changing the concepts 'rectal bleeding' to 'blood in stool', and 'weak urine stream' to 'slow urinary stream'. Other concepts were not considered relevant symptoms of the disease but rather effects of prior or ongoing treatment: erectile dysfunction; painful ejaculation; abdominal/pelvic/rectal pain; need to monitor/plan for urinary frequency; anxiety or nervousness over diagnosis/symptoms/treatment; inability to work and/or financial difficulties; and need to monitor/plan for bowel movement.

Experts suggested that a significant number of relevant concepts were not present in the model and should be candidates for addition. These included diarrhea; rectal bleeding; bone pain; fractures; spinal cord compressions; nerve compressions; access to treatment; relationship stress as a result of sexual dysfunction; general pain; fatigue; anxiety (e.g., from ambiguity of diagnosis/concern over future of disease); new urinary symptoms; ability of patients to do the things they want to do; back pain; and interference with daily activity as a result of testing and constant monitoring of PSA. Expert feedback was considered in the refinement and development of the final model.

\subsection{Step 3: Patient Interviews}

Interviewed patients ( $n=17$ ) were from 13 US states; see Table 1 for baseline characteristics. Of 17 patients with M0-CRPC, two also had other cancers (lung and bladder). Most patients were $\geq 65$ years old, and about one-third had undergone surgical castration and two-thirds were receiving LHRH/GnRH agonist therapy at the time of the study. 
Table 1 Baseline characteristics of men with non-metastatic castration-resistant prostate cancer who participated in the patient interviews

\begin{tabular}{|c|c|}
\hline Disease and demographic characteristics & Respondents $(n=17)$ \\
\hline Cancer diagnoses & $\begin{array}{l}\text { Prostate cancer: } 100 \% \\
\text { Prostate cancer }+ \text { lung cancer, } n=1 \\
\text { Prostate cancer }+ \text { bladder cancer, } n=1\end{array}$ \\
\hline Metastasized cancer to brain and bones & None reported \\
\hline Treatment for prostate cancer ${ }^{\mathrm{a}}$ & $\begin{array}{l}\text { Surgical castration, } 35 \% \\
\text { Currently receiving LHRH or GnRH agonist therapy, } 65 \% \\
\text { Qualitative data from interviews }\left(n=16^{\mathrm{b}}\right) \text { indicated: } \\
\text { Radical prostatectomy only, } n=7 \\
\text { Hormone therapy only, } n=1 \\
\text { Radical prostatectomy and hormone therapy, } n=1 \\
\text { Bilateral orchiectomy and testosterone replacement therapy, } n=1 \\
\text { Radiation ("seed implants") and hormone therapy, } n=6\end{array}$ \\
\hline PSA levels & $\begin{array}{l}\text { Stable, } 70 \% \\
\text { Rising, } 24 \% \\
\text { Do not know, } 6 \%\end{array}$ \\
\hline Age & $\begin{array}{l}\geq 65 \text { years of age, } 76 \% \\
41-64 \text { years of age, } 24 \%\end{array}$ \\
\hline Geographical spread & $\begin{array}{l}\text { Patients represent } 13 \text { different US states (southern, } n=8 \text {; western, } \\
\quad n=3 \text {; midwestern, } n=1 \text {; northeastern, } n=5 \text { ) }\end{array}$ \\
\hline
\end{tabular}

GnRH gonadotropin-releasing hormone, LHRH luteinizing hormone-releasing hormone, PSA prostate-specific antigen

${ }^{a}$ Note the discrepancy between screener findings and self-report during interviews. We hypothesize that differences are due to respondent unfamiliarity with "surgical castration" and "LHRH or GnRH agonist therapy" terminology used in the screening document and the lack of a response option for radiation therapy

${ }^{\mathrm{b}}$ One patient was not asked this question during the interview

About one-quarter of patients had rising PSA at the time of the interview; in most patients, PSA was stable (70\%).

During the interviews, patients mentioned 35 distinct symptoms, which were categorized as urinary $(n=11)$, sexual $(n=5)$, hormonal $(n=10)$, gastrointestinal $(n=7)$, or other $(n=2)$ (Table 2$)$. Symptoms mentioned most frequently were urinary (58 mentions), sexual (35 mentions), hormonal (31 mentions), and gastrointestinal (ten mentions). M0-CRPC symptoms that (a) were most frequently mentioned by patients during interviews and (b) had the highest interference ratings as reported by patients are listed in Fig. 3a, b, respectively. The most frequently mentioned symptoms were erectile dysfunction, loss of libido, urinary urgency, incontinence, and hot flashes (Fig. 3a). Patients attributed symptoms to both the disease and its treatment. Sexual and hormonal symptoms were the most commonly mentioned symptoms attributed to treatment. Median interference scores (on a scale of $0-10$, where $0=$ does not disturb and $10=$ greatly disturbs) for the most commonly reported symptoms were erectile dysfunction, 5; loss of libido, 3; urinary urgency, 6; incontinence, 3; and hot flashes, 3. Symptoms of weight gain, loss of muscle mass, inability to feel urination, and groin pain were experienced as relatively more disturbing, but these were experienced infrequently (Fig. 3b).
Overall, 23 distinct impacts were mentioned and categorized as emotional $(n=10)$, physical $(n=7)$, and socio-environmental $(n=6)$ (Table 3$)$. The most frequently mentioned impacts were emotional (43 mentions), physical (31 mentions), and socio-environmental (17 mentions). Impacts of M0-CRPC that (a) were most frequently mentioned by patients during interviews and (b) had the highest interference ratings as reported by patients are listed in Fig. 4a, b, respectively. The most frequently mentioned impacts were the need to monitor/plan for urinary frequency (reported by nine patients), interference with/restriction of daily activities (eight patients), and frustration (seven patients) or anxiety (seven patients) over diagnosis/symptoms/treatment (Fig. 4a). When asked to compare characteristics of symptoms, patients generally reported that symptom frequency, rather than severity or duration, had the most impact on their daily functioning.

Many identified impacts interfered at least moderately with patients' daily lives (Fig. 4b). However, as with symptoms, the impacts associated with the greatest interference often tended to be less frequently reported.

Complete concept saturation was not reached, defined as the point in the data collection process after which no new concepts are elicited [33]. Four new symptoms (leakage of 


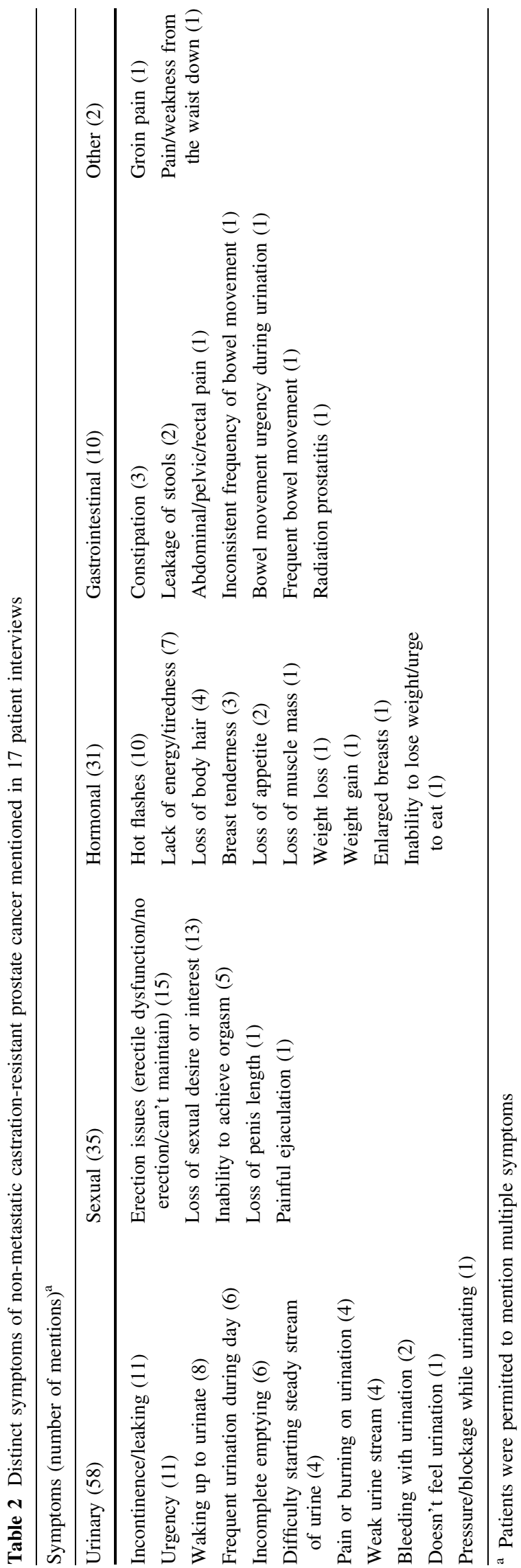

stools [interview 11], enlarged breasts [interview 10], groin pain [interview 12], and pain/weakness from the waist down [interview 13]) and one new impact (embarrassment in sexual situations [interview 15]) were mentioned in the last group of interviews (11-17). However, saturation on the most salient symptoms and impacts in the model was achieved, and it was nearly attained on the less salient symptoms and impacts, suggesting that the model sufficiently reflects patient experience with M0-CRPC.

A conceptual model of the impact of M0-CRPC and its treatments, developed from the combination of literature review and empirical data from clinicians and patients, is shown in Fig. 5. The most salient urinary symptom concepts included urgency, waking to urinate, frequent urination during day, incontinence/leaking urine, and incomplete emptying. Other salient symptom concepts were inadequate or no erection, inability to achieve orgasm, hot flashes, and lack of energy/tiredness.

In the model, impacts were categorized as 'immediate' and 'general'. Immediate impacts are more proximal to the signs/symptoms and can be directly attributed to one or more of these (e.g., if the symptom is pain, the immediate impact may be difficulty sleeping). General impacts are more distal to the signs/symptoms and more general in nature (e.g., depression, financial difficulties, etc.). Almost all of the immediate impact concepts were considered to be among those most salient (apart from need to plan/monitor bowel movements), while among the general impact concepts the most salient were stress/conflict/inability to sustain relationships with partners, friends, and family, and loss of feeling of masculinity.

As patients were typically unable to distinguish which concepts are related to their disease process and which are related to treatment they received for the disease, we cannot say for certain whether specific concepts are disease or treatment related. However, the large majority of patients surmised that most of their symptoms (and many of their impacts) occurred only after the start of treatment.

\section{Discussion}

We have developed a conceptual model that synthesizes the symptoms and impacts of men living with M0-CRPC. The model is based on information from a literature review, clinician interviews, and, most importantly, patient interviews. The literature search supports the identification of key concepts and how they are currently measured in completed and ongoing clinical trials. Interviews with experts facilitate an understanding of the clinical perspective on key concepts. Interviews with patients are considered the most important part of this three-step approach and key to understanding patients' experiences with M0- 
a

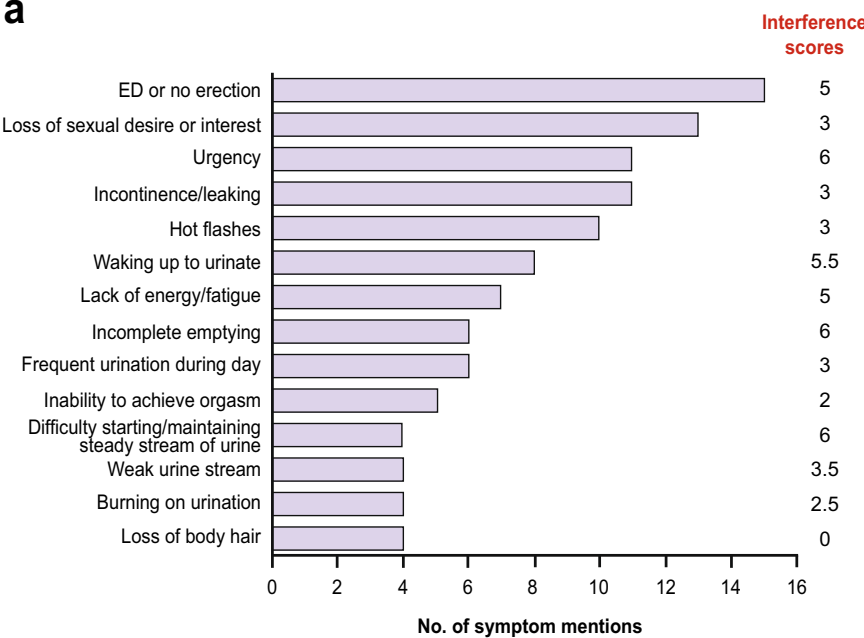

b

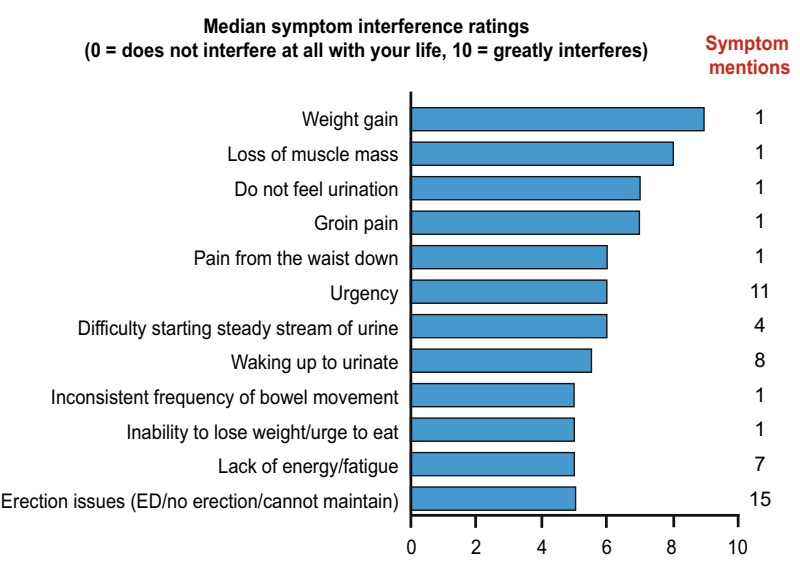

Fig. 3 Symptoms of a non-metastatic castration-resistant prostate cancer that were most frequently mentioned by patients during patient interviews and $\mathbf{b}$ castration-resistant prostate cancer that had the highest interference ratings as reported by patients. $E D$ erectile dysfunction

Table 3 Distinct impacts of non-metastatic castration-resistant prostate cancer mentioned in patient interviews

Symptoms (number of mentions) ${ }^{\mathrm{a}}$

Emotional impacts (43) $\quad$ Physical impacts (31) $\quad$ Socio-environmental impacts (17)

Anxiety/nervousness over diagnosis/

symptoms/treatment (7)

Frustration over diagnosis/symptoms/treatment (7)

Depression over diagnosis/symptoms/treatment (5)

Thoughts of death and mortality (5)

Loss of feeling of masculinity (5)

Low self-esteem (4)

Feeling of loss of control (4)

Hopelessness (3)

Feeling lonely (2)

Decreased coping ability (1)
Need to monitor/plan for urinary frequency (9)

Interference with/restriction of daily activities (8)

Poor sleep (6)

Generally feeling ill (3)

Difficulty concentrating (2)

Need to monitor/plan for bowel

movement (2)

May not be absorbing nutrients (1)
Stress or conflict with partner, friends, family (6)

Inability to work and/or financial difficulties (3)

Distrust/lack of confidence in doctor (3)

Unable to sustain relationships/social life (2)

Become more of a homebody/private (2)

Embarrassment in sexual situations (1)

a Patients were permitted to mention multiple impacts

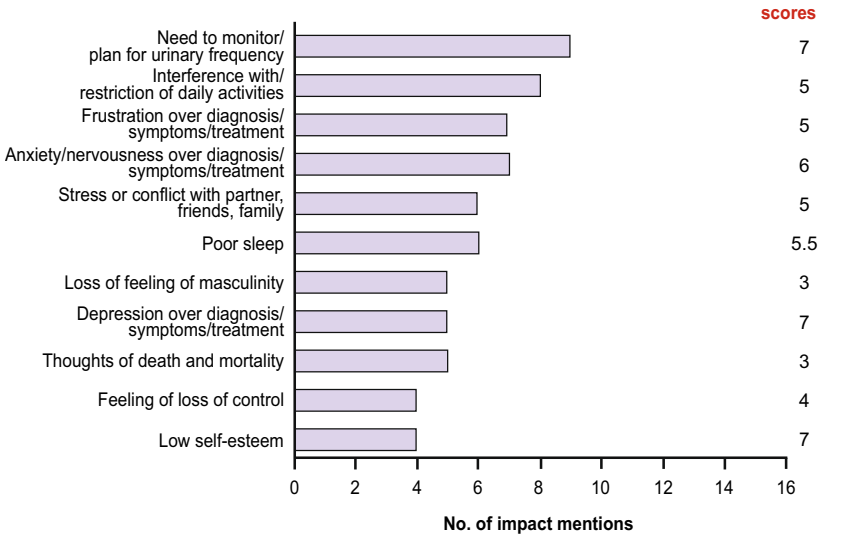

b

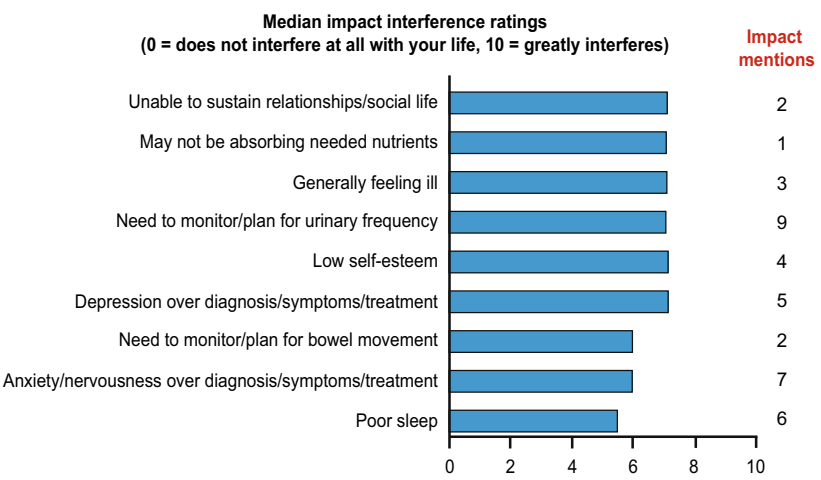

Fig. 4 a Most frequent impacts mentioned across categories and median interference score, as derived from patient interviews and $\mathbf{b}$ impact interference ratings reported by patients with non-metastatic castration-resistant prostate cancer 


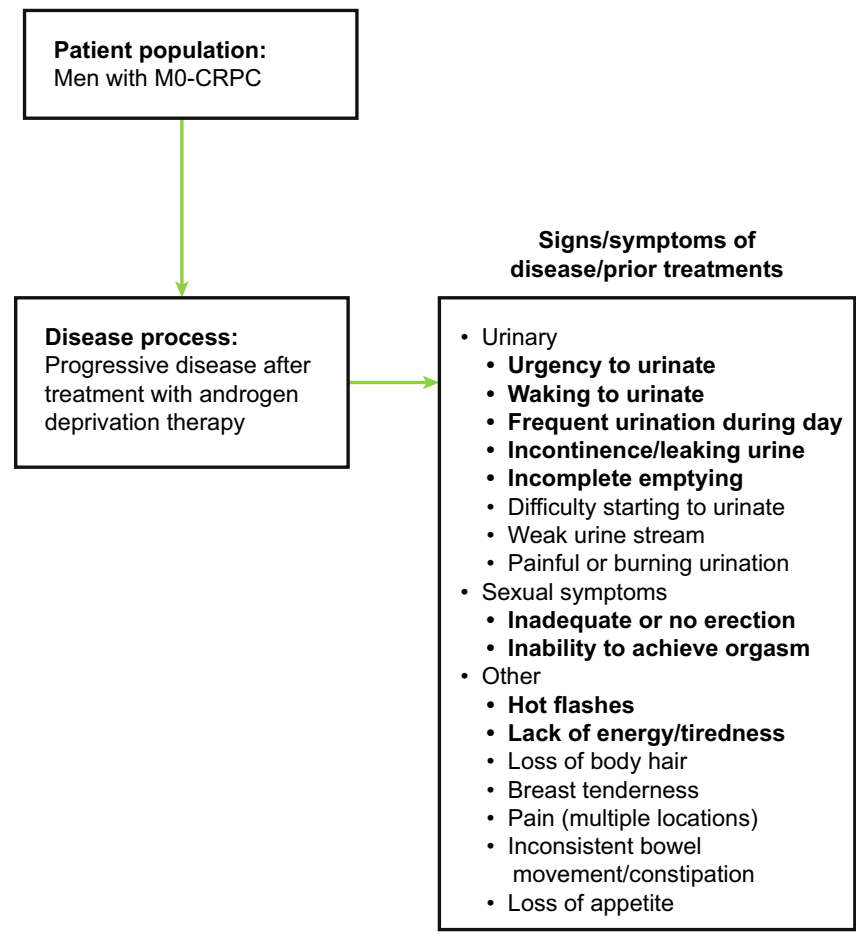

Fig. 5 Conceptual model of the impact of non-metastatic castrationresistant prostate cancer elicited from patient interviews and qualityof-life measurement identified from the literature review. Concepts in bold text are the most salient concepts. Interference with daily

CRPC and patients' perception of relevant aspects of the disease.

The patients we interviewed most often identified aspects of sexual dysfunction and urinary symptoms as exemplars of their experience, leading to interference with/ restriction of daily activities and frustration or anxiety over diagnosis/symptoms/treatment. They generally attributed these concepts to the disease itself, rather than its treatment. However, these symptoms and impacts did not seem to greatly interfere with, or disturb, patients' lives; concepts with greater effects on patients' lives were infrequently mentioned. Both the literature review and clinician interviews confirmed the salience of these sexual, urinary, and psychological concepts, but suggested that patients with M0-CRPC are bothered less by symptoms or impacts that are a consequence of disease progression than by adverse effects and consequences of prior or current treatment. Indeed, M0-CRPC has been considered a disease state, with most patients usually asymptomatic except for the adverse effects related to ADT [34]. ADT has a wellrecognized adverse event profile, including loss of libido and impotence, fatigue, vasomotor flushing, anemia, osteoporosis, and gynecomastia. In our study, $65 \%$ of patients were currently receiving ADT (LHRH or GnRH agonist therapy). Metabolic complications from such treatment have also been noted, including insulin

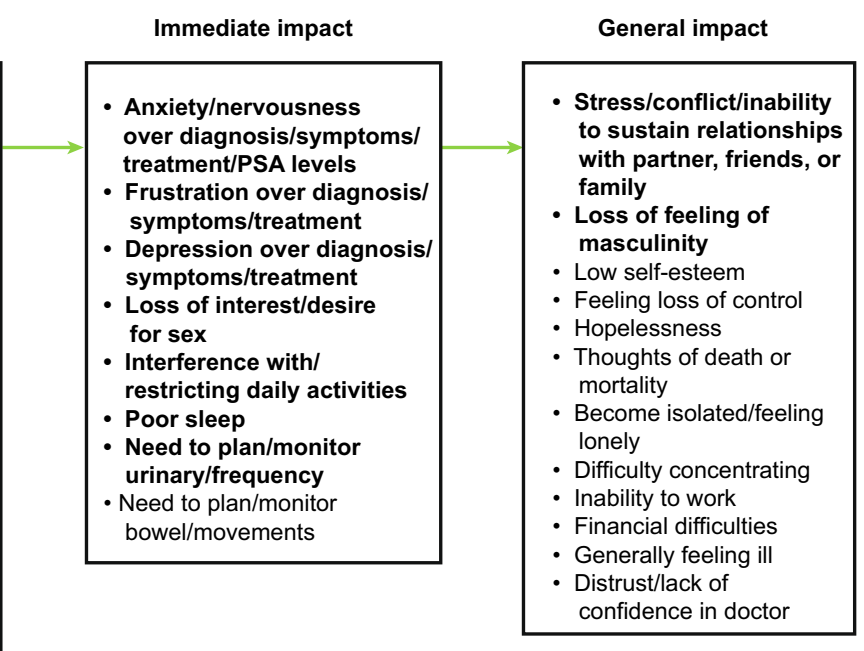

activities is a specific impact that means restriction of physical exertion and constraints on performance on household management tasks. $M 0-C R P C$ non-metastatic castration-resistant prostate cancer, $P S A$ prostate-specific antigen

resistance, obesity, and dyslipidemia, and long-term use has been associated with cardiovascular effects. Moreover, the introduction of ADT, especially in asymptomatic men, can affect HR-QoL domains, including physical function, vitality, role-physical domains, and bodily pain [35].

A recent study by Sartor et al. [13] developed a conceptual model of the impacts of advanced PCa (biochemical failure or metastatic disease) and its treatment. In the final model, patients with biochemical failure (rising PSA, detectable metastatic disease not yet present), similar to those in our study, reported urinary symptoms and sexual dysfunction as the primary symptoms. In addition, they had substantial emotional impacts, including a great deal of anxiety and depression. Emotional impacts were commonly observed in our patients (e.g., frustration or anxiety over diagnosis/symptoms/treatment). In both studies, patients who are considered largely asymptomatic experienced substantial everyday life impacts owing to treatment that were at least moderately bothersome. Symptoms in our final model that were not in the Sartor et al. [13] study included loss of body hair, pain, and certain urinary symptoms (urinary incontinence, voiding difficulties). Conversely, symptoms in the Sartor et al. [13] model but not in ours included hormone treatment-related metabolic disturbances and genital atrophy. These differences between the two models likely reflect differences in the 
criteria for inclusion of symptoms in the final model (these criteria are not explicitly stated in the Sartor et al. paper), as well as the inclusion of patients with metastatic disease in the evaluation of hormone treatment-related symptoms in the Sartor et al. [13] study.

In our study, while the most relevant and important symptoms identified by patients aligned well with those mentioned by clinicians, there was less alignment between patients and clinicians on impacts. This disparity has been previously described for localized [36] and early-stage advanced [37] PCa, where physician ratings of symptoms did not correlate well with patient self-assessments of HRQoL. In addition, it has been suggested that clinicians may focus on particularly observable symptoms rather than the broader patient experience [38].

The patient interview portion of this research implemented digital outreach to recruit patients. This approach has several advantages, primarily the ability to identify and recruit patients quickly. It also provides access to people in all areas of the USA via the internet, rather than just those who can access and travel to a research site. This webbased design may be a lower-cost alternative to traditional face-to-face interview methods. Importantly, this novel approach did not seem to decrease patients' desire to participate, as men were willing and able to participate via the internet. Moreover, we adopted a patient-centric approach to engage patients and allow them to participate in the way that worked best for them (e.g., we provided webcams to patients who did not own them to allow them to participate).

Our study was subject to several possible limitations. Firstly, diagnosis of M0-CRPC was patient reported, without confirmation from a treating clinician. The sample was recruited from men who connected to an online community, where they self-identified for $\mathrm{PCa}$, and then were screened in our study for extent of known metastasis. The awareness and knowledge of the disease and its treatment among these patients may not be representative of patients with M0-CRPC so they may not be a representative sample of men with PCa.

Another possible limitation is related to participants' PSA levels. Our study initially specified rising PSA as an inclusion criterion since our target population of M0-CRPC is characterized by rising PSA during ADT with a castration level of testosterone in the absence of clinically detectable metastatic disease. Indeed, CRPC is often identified at an early stage, when the only sign of resistance to ADT is a progressive elevation of PSA [34]. This patient population is of further interest as it the subject of a number of current trials (e.g., Safety and Efficacy Study of Enzalutamide in Patients with Non-Metastatic Castration-Resistant Prostate Cancer [PROSPER; enzalutamide; NCT02003924] [39], Study of Apalutamide [ARN-509] in Men with Non-
Metastatic Castration-Resistant Prostate Cancer [SPARTAN; apalutamide; NCT01946204] [40], and Efficacy and Safety Study of BAY 1841788 [ODM-201] in Men With High-Risk Non-Metastatic Castration-Resistant Prostate Cancer [ARAMIS; ODM-201; NCT02200614] [41]). However, this criterion was subsequently relaxed to ease recruiting by enabling inclusion of patients with stable PSA or those who did not know their PSA levels. A final possible limitation is the absence of complete concept saturation during patient interviews for concept elicitation, although this was closely approached and was achieved for the most salient symptoms and impacts.

Our data provide valuable insight into patients' experiences with M0-CRPC and may help identify the most important PROs to measure in this population. Improved understanding of patients' experiences with living with M0-CRPC expands a perspective that was previously based exclusively on literature and clinician viewpoints. Furthermore, our study argues for considering that the most relevant symptoms and impacts expressed by patients may be attributed to $\mathrm{PCa}$ therapy for M0-CRPC, more so the consequences of the disease itself. Accordingly, we suggest examining new ways of measuring sensitivity to the effects of treatment for this disease to complement existing PRO instruments.

Several concepts identified in our model, which were not specifically addressed in these other PRO instruments, include frustration over treatment, loss of body hair, low self-esteem, financial difficulties, and distrust/lack of confidence in their doctor. Additionally, low self-esteem and the need to plan for and monitor bowel movements also surfaced in our model as disease-related effects that are unaccounted for in currently used instruments. This work may help identify critical target areas for evaluation in clinical studies or guide investigators in selecting outcome variables or areas suitable for intervention.

\section{Conclusion}

We developed a conceptual model that identifies the key symptoms and impacts of M0-CRPC from the patient perspective. The symptoms and impacts most frequently experienced by patients were typically not those with the greatest effects on their lives. Those symptoms and impacts with the greatest consequences appeared to be related to treatment, which included surgery, radiotherapy, and LHRH/GnRH agonist therapy, rather than to the disease itself.

This research may help stimulate discussion about which concepts should be reflected in study endpoints and PRO tools to provide more relevant and comprehensive coverage of the patient's experience with M0-CRPC. 
Acknowledgements Thomas Lavelle of Bioscript Science provided assistance with writing and revising the draft manuscript, based on detailed discussion and feedback from all authors. Editorial assistance was provided by Stephanie Rippon, Jane Beck, and Lauren Smith of Complete HealthVizion, all funded by the research sponsors. We gratefully thank the investigators and patients for their participation in the study.

Author contributions All authors contributed to the concept and design of the study. Shevani Naidoo and Stefan Holmstrom contributed to the collection and/or assembly of data. Erin L. Tomaszewski, Pierre Moise, Robert N. Krupnick, Jared Downing, and Margaret Meyer contributed to the data analysis and interpretation. All authors contributed to the drafting of the manuscript and its critical revision for important intellectual content. All authors approved the final manuscript. All study documents were reviewed and approved by QuintilesIMS' Institutional Review Board, and all patients participating in this study provided signed informed written consent.

\section{Compliance with Ethical Standards}

Funding This study was funded by Astellas Pharma, Inc. and Medivation, Inc. (which was acquired by Pfizer, Inc. in September 2016), the co-developers of enzalutamide.

Conflict of interest Pierre Moise, Robert N. Krupnick, Jared Downing, and Margaret Meyer are employees of QuintilesIMS, which received funding from Astellas to conduct the research/analyses reported. At the time this research was conducted and during the development of this publication, Erin L. Tomaszewski was an employee of QuintilesIMS. Shevani Naidoo and Stefan Holmstrom are employees of Astellas Pharma, Inc.

Open Access This article is distributed under the terms of the Creative Commons Attribution-NonCommercial 4.0 International License (http://creativecommons.org/licenses/by-nc/4.0/), which permits any noncommercial use, distribution, and reproduction in any medium, provided you give appropriate credit to the original author(s) and the source, provide a link to the Creative Commons license, and indicate if changes were made.

\section{References}

1. Sternberg CN, Fizazi K, Saad F, Shore N, Heidenreich A, Hirmand $\mathrm{M}$, et al. PROSPER: a phase III study of enzalutamide in non-metastatic (M0) castration-resistant prostate cancer (CRPC) patients [poster no. 802TiP]. ESMO Congress; 26-30 Sep 2014, Madrid.

2. Hotte SJ, Saad F. Current management of castrate-resistant prostate cancer. Curr Oncol. 2010;17(Suppl 2):S72-9.

3. Hong JH, Kim IY. Nonmetastatic castration-resistant prostate cancer. Korean J Urol. 2014;55(3):153-60.

4. Eton DT, Lepore SJ. Prostate cancer and health-related quality of life: a review of the literature. Psychooncology. 2002;11(4):307-26.

5. Merseburger AS, Bellmunt J, Jenkins C, Parker C, Fitzpatrick JM. Perspectives on treatment of metastatic castration-resistant prostate cancer. Oncologist. 2013;18(5):558-67.

6. Sullivan PW, Mulani PM, Fishman M, Sleep D. Quality of life findings from a multicenter, multinational, observational study of patients with metastatic hormone-refractory prostate cancer. Qual Life Res. 2007;16(4):571-5.

7. US Department of Health and Human Services, Food and Drug Administration, Center for Drug Evaluation and Research (CDER), Center for Biologics Evaluation and Research (CBER), Center for Devices and Radiological Health (CDRH). Guidance for industry. Patient-reported outcomes measures: use in medical product development to support labeling claims. http://www.fda gov/downloads/Drugs/GuidanceComplianceRegulatoryInformation/ Guidances/UCM193282.pdf. Accessed 27 Jan 2017.

8. Esper P, Mo F, Chodak G, Sinner M, Cella D, Pienta KJ. Measuring quality of life in men with prostate cancer using the Functional Assessment of Cancer Therapy-Prostate instrument. Urology. 1997;50(6):920-8.

9. van Andel G, Bottomley A, Fosså SD, Efficace F, Coens C, Guerif S, et al. An international field study of the EORTC QLQPR25: a questionnaire for assessing the health-related quality of life of patients with prostate cancer. Eur $\mathrm{J}$ Cancer. 2008;44(16):2418-24.

10. European Medicines Agency. Reflection paper on the use of patient reported outcome (PRO) measures in oncology studies. http://www. ema.europa.eu/docs/en_GB/document_library/Scientific_guideline/ 2014/06/WC500168852.pdf. Accessed 27 Jan 2017.

11. Rothman ML, Beltran P, Cappelleri JC, Lipscomb J, Teschendorf B, the Mayo/FDA Patient-Reported Outcomes Consensus Meeting Group. Patient-reported outcomes: conceptual issues. Value Health. 2007;10(Suppl 2):S66-75.

12. Eton DT, Shevrin DH, Beaumont J, Victorson D, Cella D. Constructing a conceptual framework of patient-reported outcomes for metastatic hormone-refractory prostate cancer. Value Health. 2010;13(5):613-23.

13. Sartor O, Flood E, Beusterien K, Park J, Webb I, MacLean D, et al. Health-related quality of life in advanced prostate cancer and its treatments: biochemical failure and metastatic disease populations. Clin Genitourin Cancer. 2015;13(2):101-12.

14. Patrick DL, Burke LB, Gwaltney CJ, Leidy NK, Martin ML, Molsen E, et al. Content validity-establishing and reporting the evidence in newly developed patient-reported outcomes (PRO) instruments for medical product evaluation: ISPOR PRO Good Research Practices Task Force Report: part 1-eliciting concepts for a new PRO instrument. Value Health. 2011;14(8):967-77.

15. Patrick DL, Burke LB, Gwaltney CJ, Leidy NK, Martin ML, Molsen E, et al. Content validity-establishing and reporting the evidence in newly developed patient-reported outcomes (PRO) instruments for medical product evaluation: ISPOR PRO Good Research Practices Task Force Report: part 2-assessing respondent understanding. Value Health. 2011;14(8):978-88.

16. MediGuard.org. Medication monitoring made simple. https:// www.mediguard.org/. Accessed 27 Jan 2017.

17. Aning JJ, Wassersug RJ, Goldenberg SL. Patient preference and the impact of decision-making aids on prostate cancer treatment choices and post-intervention regret. Curr Oncol. 2012;19(Suppl 3):S37-44.

18. Befort CA, Zelefsky MJ, Scardino PT, Borrayo E, Giesler RB, Kattan MW. A measure of health-related quality of life among patients with localized prostate cancer: results from ongoing scale development. Clin Prostate Cancer. 2005;4(2):100-8.

19. Bokhour BG, Clark JA, Inui TS, Silliman RA, Talcott JA. Sexuality after treatment for early prostate cancer: exploring the meanings of "erectile dysfunction". J Gen Intern Med. 2001;16(10):649-55.

20. Chang P, Szymanski KM, Dunn RL, Chipman JJ, Litwin MS, Nguyen PL, et al. Expanded prostate cancer index composite for clinical practice: development and validation of a practical health related quality of life instrument for use in the routine clinical care of patients with prostate cancer. J Urol. 2011;186(3):865-72. 
21. Clark JA, Inui TS, Silliman RA, Bokhour BG, Krasnow SH, Robinson RA, et al. Patients' perceptions of quality of life after treatment for early prostate cancer. J Clin Oncol. 2003;21(20):3777-84.

22. Ellison JS, He C, Wood DP. Stratification of postprostatectomy urinary function using expanded prostate cancer index composite. Urology. 2013;81(1):56-60.

23. FACIT.org. FAPSI-8: for patients with prostate cancer; A FACTProstate Symptom Index (a subset of FACT-P containing 8 items). http://www.facit.org/FACITOrg/Questionnaires. Accessed 27 Jan 2017.

24. Gibbons EJ, Morris C, Fitzpatrick R. A structured review of patient-reported outcome measures (PROMs) for prostate cancer. Oxford: Nuffield Department of Population Health; 2009.

25. Katz A. What happened? Sexual consequences of prostate cancer and its treatment. Can Fam Physician. 2005;51:977-82.

26. Kemmler G, Holzner B, Kopp M, Dünser M, Margreiter R, Greil $\mathrm{R}$, et al. Comparison of two quality-of-life instruments for cancer patients: the Functional Assessment of Cancer Therapy-General and the European Organization for Research and Treatment of Cancer Quality of Life Questionnaire-C30. J Clin Oncol. 1999;17(9):2932-40.

27. Morton GC, Loblaw DA, Chung H, Tsang G, Sankreacha R, Deabreu A, et al. Health-related quality of life after single-fraction high-dose-rate brachytherapy and hypofractionated external beam radiotherapy for prostate cancer. Int J Radiat Oncol Biol Phys. 2011;80(5):1299-305.

28. Potosky AL, Reeve BB, Clegg LX, Hoffman RM, Stephenson RA, Albertsen PC, et al. Quality of life following localized prostate cancer treated initially with androgen deprivation therapy or no therapy. J Natl Cancer Inst. 2002;94(6):430-7.

29. Rnic K, Linden W, Tudor I, Pullmer R, Vodermaier A. Measuring symptoms in localized prostate cancer: a systematic review of assessment instruments. Prostate Cancer Prostatic Dis. 2013;16(2):111-22.

30. The University of Michigan. EPIC-26. The Expanded Prostate Cancer Index Composite. Short Form. https://medicine.umich. edu/sites/default/files/content/downloads/EPIC\%20Short\%20Form_ 0.pdf. Accessed 27 Jan 2017.

31. Wei JT, Dunn RL, Sandler HM, McLaughlin PW, Montie JE, Litwin MS, et al. Comprehensive comparison of health-related quality of life after contemporary therapies for localized prostate cancer. J Clin Oncol. 2002;20(2):557-66.

32. Yount S, Cella D, Banik D, Ashraf T, Shevrin D. Brief assessment of priority symptoms in hormone refractory prostate cancer: the FACT Advanced Prostate Symptom Index (FAPSI). Health Qual Life Outcomes. 2003;1:69.

33. Rothman M, Burke L, Erickson P, Leidy NK, Patrick DL, Petrie $\mathrm{CD}$. Use of existing patient-reported outcome (PRO) instruments and their modification: the ISPOR good research practices for evaluating and documenting content validity for the Use of Existing Instruments and their Modification PRO Task Force Report. Value Health. 2009;12(8):1075-83.

34. Tombal B. Non-metastatic CRPC and asymptomatic metastatic CRPC: which treatment for which patient? Ann Oncol. 2012;23(Suppl 10):x251-8.

35. Ahmadi H, Daneshmand S. Androgen deprivation therapy for prostate cancer: long-term safety and patient outcomes. Patient Relat Outcome Meas. 2014;5:63-70.

36. Sonn GA, Sadetsky N, Presti JC, Litwin MS. Differing perceptions of quality of life in patients with prostate cancer and their doctors. J Urol. 2009;182(5):2296-302.

37. Litwin MS, Lubeck DP, Henning JM, Carroll PR. Differences in urologist and patient assessments of health related quality of life in men with prostate cancer: results of the CaPSURE database. J Urol. 1998;159(6):1988-92.

38. Payne H, Pearcy R. Symptoms and health-related quality of life in castration-resistant prostate cancer: the patient's perspective. J Mens Health. 2013;9(1):9-16.

39. Hussain M, Fizazi K, Saad F, Shore ND, Heidenreich A, Hirmand $M$, et al. PROSPER: a phase 3 study of enzalutamide in nonmetastatic (M0) castration-resistant prostate cancer (CRPC) patients [abstract no. TPS5094]. J Clin Oncol. 2014;32(Suppl):5s.

40. Smith MR, Antonarakis ES, Ryan CJ, Berry WR, Shore ND, Liu $\mathrm{G}$, et al. Phase 2 study of the safety and antitumor activity of apalutamide (ARN-509), a potent androgen receptor antagonist, in the high-risk nonmetastatic castration-resistant prostate cancer cohort. Eur Urol. 2016;70(6):963-70.

41. Fizazi K, Albiges L, Loriot Y, Massard C. ODM-201: a newgeneration androgen receptor inhibitor in castration-resistant prostate cancer. Expert Rev Anticancer Ther. 2015;15(9): $1007-17$. 